\title{
Short-term outcomes following minimally invasive and open esophagectomy: a population-based study from Finland and Sweden
}

\section{Running head: Short-term outcomes of MIE and OE}

Authors: *Joonas H Kauppila, $\mathrm{MD}, \mathrm{PhD}^{1,2}$, *Olli Helminen $\mathrm{MD}, \mathrm{PhD}^{2,3}$, Ville Kytö MD, $\mathrm{PhD}^{4}$, Jarmo Gunn MD, $\mathrm{PhD}^{5}$, Jesper Lagergren $\mathrm{MD}, \mathrm{PhD}^{1,6}$, Eero Sihvo $\mathrm{MD}, \mathrm{PhD}^{3}$

*Equal contribution as first author

${ }^{1}$ Upper Gastrointestinal Surgery, Department of Molecular Medicine and Surgery, Karolinska Institutet, Karolinska University Hospital, 17176 Stockholm, Sweden

${ }^{2}$ Cancer and Translational Medicine Research Unit, Medical Research Center Oulu, University of Oulu and Oulu University Hospital, Oulu, Finland

${ }^{3}$ Department of Surgery, Central Finland Central Hospital, 40620 Jyväskylä, Finland

${ }^{4}$ Heart Center, Turku University Hospital, Turku, Finland. Research Center of Applied and Preventive Cardiovascular Medicine, University of Turku, Turku, Finland

${ }^{5}$ Heart Center, Turku University Hospital, Turku, Finland Department of Surgery, Faculty of Medicine, University of Turku, Turku, Finland

${ }^{6}$ Division of Cancer Studies, King's College London and Guy's and St Thomas' NHS

Foundation Trust, London, United Kingdom.

Disclosure: The authors declare no conflicts of interest.

Contact information of the corresponding author:

Eero Sihvo, MD, PhD

Department of Surgery

Central Finland Central Hospital

Keskussairaalantie 19, 40620 Jyväskylä, FINLAND

tel: +358142691811

e-mail: eero.sihvo@ksshp.fi

Abstract 247 words, text 2355 words 


\section{Synopsis}

In this population-based study, we compare short-term outcomes following minimally invasive $(n=217)$ and open $(n=1397)$ esophagectomy. Adjusted 90-day mortality, 30-day readmission rate, and median hospital stay were lower after minimally invasive esophagectomy, supporting its use as a primary surgical approach. 
Background: Population-based studies comparing minimally invasive and open esophagectomy relative to 90-day postoperative mortality are needed. The aim of this study was to compare short-term outcomes following these two techniques for esophageal cancer.

Methods: Patients undergoing minimally invasive $(n=217)$ or open $(n=1397)$ esophagectomy for esophageal cancer between 2007 and 2014 were identified from nationwide complete registries in Finland and Sweden. The primary outcome was 90-day mortality. Secondary outcomes were 30-day mortality, length of hospital stay, and 30- and 90-day readmission rate. Results were adjusted for age, sex, comorbidity, tumor histology, surgery year, and country.

Results: 90-day mortality rates were $4.1 \%$ ( $\mathrm{n}=9$ out of 217 ) for minimally invasive and $6.8 \%$ ( $\mathrm{n}=95$ out of 1397) for open esophagectomy, and 90-day mortality was halved after minimally invasive esophagectomy (adjusted HR 0.49, 95\% CI 0.24-0.99). There was no difference in 30day mortality (adjusted HR $0.87,95 \%$ CI $0.29-2.66$ ). Median hospital stay was 15 days for minimally invasive and 16 days for open esophagectomy (adjusted $\beta-0.17$, standard error 0.08 , $\mathrm{P}=0.030$ ). The 30 -day readmission rates were $8.9 \%$ after minimally invasive and $12.0 \%$ after open esophagectomy (adjusted HR 0.57, 95\% CI 0.34-0.94), while the 90-day readmission rates were $28.8 \%$ and $33.6 \%$ respectively, without a statistically significant difference (adjusted HR $0.82,95 \%$ CI 0.61-1.10).

Conclusions: This population-based study from Finland and Sweden revealed lower 90-day mortality, shorter hospital stay and lower 30-day readmission rate after minimally invasive esophagectomy compared to open esophagectomy for esophageal cancer. These findings support the use of minimally invasive approaches.

Keywords: Esophageal cancer, minimally invasive surgery, esophagectomy, short-term mortality 


\section{Introduction}

Esophageal cancer is the sixth leading cause of cancer death worldwide, ${ }^{1}$ and surgery offers the best chance for cure in early or locally advanced disease. ${ }^{2}$ Esophagectomy is an extensive operation with considerable risks of morbidity and mortality. To reduce complications, the use of minimally invasive esophagectomy (MIE) has increased during the last years. ${ }^{3}$

One randomized study and several single-center series suggest that MIE reduces postoperative morbidity, shortens hospital stay, and improves patient satisfaction compared to open esophagectomy (OE) without compromising the long-term oncological outcomes. ${ }^{4-10}$ However, these findings originate from high-volume surgeons at high-volume centers, which do not necessarily reflect the results at the population level. Two population-based national studies, one from the United Kingdom and one from Japan, found no differences in 30-day mortality or morbidity between MIE and OE, but the reoperation rate within 30 days of surgery was higher after MIE in both studies. ${ }^{11,12}$ Similarly, when compared to OE, MIE was not associated with lower rates of complications or 30-day mortality, but rather with a higher reoperation rate in a study from the United States. ${ }^{13}$ The 30-day mortality rate after esophagectomy has been around $4 \%$ to $5 \%$ in several European countries and the United States, ${ }^{11,14}$ but risk of mortality persists beyond the traditional 30-day mortality assessment. ${ }^{15}$ Therefore, 90 -day mortality is increasingly considered to be a better short-term outcome measure for major surgery, ${ }^{14-16}$ yet no populationbased study comparing the 90-day outcomes between MIE and OE has been reported. Lower 90day mortality has been reported after hybrid procedure in a nationwide French study. ${ }^{17}$

Here we conduct a population-based and nationwide study in Finland and Sweden with the aim to test whether MIE or OE provide better 90-day outcomes following esophagectomy for esophageal cancer in an unselected cohort of patients. 


\section{Methods}

\section{Design}

All patients who underwent MIE or OE for cancer of the esophagus or gastroesophageal junction in Finland or Sweden from 2007 through 2014 were eligible for this population-based nationwide cohort. The two approaches were compared regarding 90-day all-cause mortality as the main outcome, while 30-day all-cause mortality, length of hospital stay, and readmission rates within 30 and 90 days of surgery were secondary outcomes. The National Institute for Health and Welfare of Finland (permissions no: THL/143/5.05.00/2015 and THL/1349/5.05.00/2015) and Regional Ethical Review Board in Stockholm, Sweden (DNR2015/1916-31/1 and 2016/584-32), approved the study.

\section{Data collection}

All residents in both countries have unique and immutable 10- or 11-digit national registration numbers, which allows for a high identification rate of patients from hospital records, administrative databases, and national health data registries and makes the unification of these databases reliable. In this study, patients who underwent esophagectomy for esophageal cancer between January 1, 2007 and October 2, 2014 were retrospectively identified from the Care Register for Healthcare in Finland and the Cancer Registry and Patient Registry in Sweden. All patients were followed up until 90 days post-surgery, death, or emigration. Follow-up ended December 31, 2014. Mortality data was linked for each patient individually from Statistics Finland and from the Swedish Causes of Death Registry. These registries had complete nationwide coverage during the study period and reporting to the registries is compulsory in Finland and Sweden. The coverage of the Finnish Hospital Discharge Registry and the Swedish 
Patient Registry are also high because of the legislative obligation of reporting and the relationship of reporting and hospital funding. The Finnish Cancer Registry has a nationwide coverage over 99\%, ${ }^{18}$ and the Swedish Cancer Registry has at least 98\% nationwide coverage for all esophageal cancer. ${ }^{19}$ The Finnish Hospital Discharge Registry and Care Register for Healthcare and the Swedish Patient Registry were also used to obtain data on patients' medical comorbidities, readmissions, and lengths of hospital stay. Data recorded in each of these registers are reliable in both Finland ${ }^{20}$ and Sweden, ${ }^{21}$ and the complete reporting of the discharge codes is encouraged by its relationship to hospital funding. Furthermore, all hospitals are obligated by legislation to report every inpatient treatment period and all outpatient contacts annually. Each healthcare contact is provided with baseline data (including age, sex, and speciality of healthcare contact), data on length of hospital stay, performed procedures, as well as discharge diagnoses and complications. Charlson comorbidity index was calculated from diagnoses in the registries in 2004-2014, according to a validated algorithm, ${ }^{22}$ and excluding esophageal cancer. Gastric cancer was also excluded from the comorbidity index because the diagnoses of distal esophageal and proximal gastric cancer can overlap in the diagnostic phase. ${ }^{19}$ Standard procedures in Finland and Sweden during the study period were either Ivor-Lewis esophagectomy with intrathoracal anastomosis, or McKeown procedure with cervical anastomosis both in MIE and OE, with the majority performed according to Ivor-Lewis method. ${ }^{23,24}$

\section{Statistical analysis}

Baseline characteristics were analysed with Chi-square or ANOVA tests as appropriate. KaplanMeier survival curves were calculated according to the life table method to visualize the crude all-cause mortality and readmission rates within 90 days of surgery. Cox regression was used for 
calculating the relative risks of mortality and readmissions, and linear regression was used in analyses of length of hospital stay (logarithmically transformed due to skewness). OE was used as the reference group in all these analyses. The regression models were adjusted for six covariates that were considered as potential confounding factors: age (continuous variable), sex (male or female), Charlson comorbidity score ( $0,1,2,3-5$, or $\geq 6$ ), histological tumor type (adenocarcinoma or squamous cell carcinoma), year of the surgery (each year between 2007 and 2014), and country (Finland or Sweden). As MIE was conducted only in higher volume hospitals, adjustment for hospital volume was not feasible. Instead, a subgroup analysis was performed with the previously mentioned adjustments for patients operated on only in hospitals performing both MIE and OE. Patients who died during hospital admission for esophagectomy were censored from the length of hospital stay analysis. Patients who died within the first 30 days of surgery were censored from the analysis of 30-day readmissions, and patients who died during 90 days were censored from 90-day readmissions. All analyses were conducted using the statistical software SAS version 9.4 (SAS Institute Inc., Cary, NC, USA). 


\section{Results}

\section{Patients}

Between January 2007 and October 2014, 1614 patients underwent esophagectomy for esophageal cancer in Finland or Sweden, including 217 (13\%) with MIE and 1397 (87\%) with OE (Table 1). Among the 35 hospitals conducting esophagectomies during the study period, a total of 11 performed both MIE $(n=217)$ and $\mathrm{OE}(\mathrm{n}=873)$. The mean age at surgery was 64.8 years. Most patients (53\%) had no reported comorbidity (Charlson comorbidity score of 0 ) and adenocarcinoma histology (74\%). Patients in the MIE group had less comorbidity than those in the OE group ( $65 \%$ vs $51 \%$ had no comorbidity, $P=0.006)$. The proportion of patients with MIE increased during the study period, but otherwise there were no major baseline differences between comparison groups (Table 1).

\section{0-day mortality}

The 90 -day mortality rate was $4.1 \%$ in the MIE group and $6.8 \%$ in the OE group (Fig. 1), and the adjusted risk of 90-day mortality was reduced by $51 \%$ after MIE compared to OE (HR 0.49, $95 \%$ CI 0.24-0.99) (Table 2). In a subgroup analysis of patients operated in hospitals performing both MIE and OE only, the 90 -day mortality rate was $4.1 \%$ in the MIE group and $6.0 \%$ in the OE group, with an adjusted HR of 0.54 (95\% CI 0.24-1.18) comparing MIE with OE.

\section{0-day mortality}

The 30-day mortality rate for all patients was $1.8 \%$ following MIE and 1.9\% for OE (Fig. 1), with no difference in risk comparing MIE and OE (adjusted HR 0.87, 95\% CI 0.29-2.66) (Table 2). 
The subgroup analysis of patients operated in hospitals conducting both MIE and OE showed similar findings with an adjusted HR of 0.77 (95\% CI 0.22-2.67).

\section{Hospital stay}

The median length of hospital stay was 15 days after MIE and 16 days after OE, and the crude linear regression analysis showed no difference between the groups. However, the adjusted analysis revealed a reduced length of hospital stay after MIE compared to OE $(P=0.03)$ (Table 3). The median hospital stay in the subgroup analysis after surgery in hospitals performing both MIE and OE was slightly shorter after MIE (15 days) than after OE (17 days), and the difference was statistically significant in the adjusted analysis $(P=0.01)$.

\section{Readmissions after surgery}

Within the first 30 postoperative days, $8.9 \%$ of patients were readmitted after MIE, compared to $12.0 \%$ after OE. The adjusted HR for 30-day readmission was reduced after MIE compared to OE (HR 0.57, 95\% CI 0.34-0.94) (Table 4), and the proportion of patients readmitted within 90 postoperative days was $28.8 \%$ after MIE and $33.6 \%$ after OE, but the adjusted analysis showed no statistically significant difference (HR 0.82, 95\% CI 0.61-1.10) (Table 4).

In the subgroup analysis of patients operated on in hospitals conducting both MIE and OE, 8.9\% of the MIE patients and $9.9 \%$ of the OE patients were readmitted within the first 30 postoperative days, and the adjusted HR was 0.71 (95\% CI 0.40-1.27). The HR for 90-day readmission was similar to that found in the main analysis (HR 0.87, 95\% CI 0.62-1.22). 


\section{Discussion}

The results of the present study suggest that MIE is followed by a lower 90 -day mortality rate compared to OE for esophageal cancer. MIE was also associated with a slightly shorter length of hospital stay and lower 30-day readmission rate compared to OE.

The main strength of this study is the population-based design with complete nationwide data from two Nordic countries including complete follow-up of all patients. This design counteracted selection bias, which is otherwise a frequent issue in retrospective studies. The design also provides an opportunity to assess the differences between MIE and OE in general practice, which might not be the case in clinical trials or reports from single high-volume centers. On the other hand, confounding is a limitation of the observational study design. We took this into account by adjusting for various potential confounding variables, such as age, comorbidity, and histology. The lack of information about tumor stage could also introduce confounding, because the MIE group might include more early stage tumors. However, short-term mortality rates are highly specific to surgery-related deaths and not tumor stage in esophageal cancer surgery, ${ }^{16,25}$ so the influence of tumor stage should be limited. Another concern is the lack of data on complications. However, we used length of hospital stay and readmission rates as proven reliable proxies for complications. ${ }^{26}$ Due to the lack of separate coding, the register data could not differentiate totally minimally invasive surgery from a hybrid procedure. This fact and the inclusion of the learning curve phase of MIE in both countries should dilute the beneficial effects of MIE rather than favour minimally invasive surgery. On the other hand, MIE is more likely to be conducted by high-volume centers and surgeons and confounding by surgeon or hospital volume could be a problem. ${ }^{27}$ However, the subgroup analyses of only hospitals conducting both MIE and OE 
showed similar results to that found in the main analysis, suggesting that the observed risk reduction by MIE versus OE was not due to bias by surgeon or hospital volume. Finally, chance could have influenced the results, particularly in the subgroup analyses, but chance errors are unlikely to be explanations for the identified positive associations.

Previously a French population-based study reported lower 90-day mortality after hybrid procedure suggesting beneficial effects of laparoscopic gastric mobilization compared to laparotomy during esophagectomy. ${ }^{17}$ The current study is, to our knowledge, the first to compare totally MIE and OE for esophageal cancer using 90-day mortality at a national level. The findings presented here of decreased 90-day but not 30-day mortality following MIE compared to OE support the notion that 90-day mortality might be a better short-term outcome measure than 30-day mortality in esophageal cancer surgery. ${ }^{15}$ Previous studies have suggested that MIE reduces postoperative complications (especially pulmonary complications),,${ }^{9,2}$ which may explain the reduced mortality after MIE identified in the present study. The similar 30-day mortality rates are in line with the findings seen in previous population-based studies. ${ }^{11,12}$ 90-day mortality rates were substantially higher than 30-day mortality rates for MIE (1.8\% vs 4.1\%) and even more so for OE (1.9\% vs 6.8\%) in this study. Similar increases have been reported in other large studies from the United States, France, and Sweden. ${ }^{14,15,17}$ The deaths occurring between 30 and 90 days of surgery are usually related to the operation, with anastomotic leakage being the single most common cause of mortality. ${ }^{16}$ Complications are treated intensively and patients could survive beyond the initial 30 days due to improved perioperative care. 
The median length of hospital stay in the current study was shorter after MIE compared to OE. No such difference in length of stay has been identified in studies from Japan and England. ${ }^{12}{ }^{11}$ However, in a nationwide French study, hospital stay was reduced by two days after laparoscopic gastric mobilization, ${ }^{17}$ and a randomized study comparing MIE and OE conducted in highvolume centers suggested a reduction of hospital stay by three days after MIE. ${ }^{9}$ Moreover, a US database study suggested a reduction of hospital stay by one day, ${ }^{29}$ and similar results were found in a Finnish single-center study. ${ }^{24}$ The reduction in the present study is therefore well in line with previous literature.

In the present study, MIE was associated with fewer readmissions at 30 days, while the rates of readmissions were similar between the surgical approaches within 90 days of surgery. The lower rate of readmissions at 30 days after MIE could be explained by the better recovery and less pain observed after MIE. ${ }^{30,31}$ Previous studies have reported 7-13\% 30-day readmission rates without a difference between the approaches. ${ }^{11,29}$ No 90 -day readmission rates have been previously reported at the national level. The high 90-day readmission rate in the present study could be due to cancer management, i.e. the start of perioperative chemotherapy.

The findings in this study could have clinical and research implications. An important foundation for the comparison of MIE and $\mathrm{OE}$ is the recent finding in the randomized TIME-trial showing equal overall and disease-free survival between MIE and OE. ${ }^{10}$ Therefore, the observed reduction in early mortality, shorter hospital stay, and lower readmission rates suggests MIE as a primary surgical method for esophageal cancer. Whether MIE could be safely used for patients 
medically unfit for open esophageal surgery with acceptable short-term mortality and morbidity remains to be studied.

In conclusion, this population-based study from the entirety of Finland and Sweden indicates lower 90-day mortality, shorter hospital stay and reduced rate of readmissions after MIE compared to OE. These findings support the use of MIE in esophageal cancer surgery.

\section{Acknowledgements:}

This study was supported by grant from Sigrid Juselius Foundation (JHK), Orion Research Foundation (JHK), Swedish Research Council (JL) and the Swedish Cancer Society (JL). 


\section{References}

1. Global Burden of Disease Cancer Collaboration, Fitzmaurice C, Allen C, et al. Global, Regional, and National Cancer Incidence, Mortality, Years of Life Lost, Years Lived With Disability, and Disability-Adjusted Life-years for 32 Cancer Groups, 1990 to 2015: A Systematic Analysis for the Global Burden of Disease Study. JAMA Oncol 2017;3:524-548.

2. Lordick F, Mariette C, Haustermans K, et al. Oesophageal cancer: ESMO Clinical Practice Guidelines for diagnosis, treatment and follow-up. Ann Oncol 2016;27:v50-v57.

3. Lazzarino AI, Nagpal K, Bottle A, et al. Open versus minimally invasive esophagectomy: trends of utilization and associated outcomes in England. Ann Surg 2010;252:292-298.

4. Luketich JD, Alvelo-Rivera M, Buenaventura PO, et al. Minimally invasive esophagectomy: outcomes in 222 patients. Ann Surg 2003;238:486-94; discussion 494-5.

5. Pennathur A, Luketich JD. Minimally invasive esophagectomy: short-term outcomes appear comparable to open esophagectomy. Ann Surg 2012;255:206-207.

6. Luketich JD, Pennathur A, Awais O, et al. Outcomes after minimally invasive esophagectomy: review of over 1000 patients. Ann Surg 2012;256:95-103.

7. Palazzo F, Rosato EL, Chaudhary A, et al. Minimally invasive esophagectomy provides significant survival advantage compared with open or hybrid esophagectomy for patients with cancers of the esophagus and gastroesophageal junction. J Am Coll Surg 2015;220:672-679. 
8. Sihag S, Wright CD, Wain JC, et al. Comparison of perioperative outcomes following open versus minimally invasive Ivor Lewis oesophagectomy at a single, high-volume centre. Eur J Cardiothorac Surg 2012;42:430-437.

9. Biere SS, van Berge Henegouwen MI, Maas KW, et al. Minimally invasive versus open oesophagectomy for patients with oesophageal cancer: a multicentre, open-label, randomised controlled trial. Lancet 2012;379:1887-1892.

10. Straatman J, van der Wielen N, Cuesta MA, et al. Minimally Invasive Versus Open Esophageal Resection: Three-year Follow-up of the Previously Reported Randomized Controlled Trial: the TIME Trial. Ann Surg 2017.

11. Mamidanna R, Bottle A, Aylin P, et al. Short-term outcomes following open versus minimally invasive esophagectomy for cancer in England: a population-based national study. Ann Surg 2012;255:197-203.

12. Takeuchi H, Miyata H, Ozawa S, et al. Comparison of Short-Term Outcomes Between Open and Minimally Invasive Esophagectomy for Esophageal Cancer Using a Nationwide Database in Japan. Ann Surg Oncol 2017.

13. Sihag S, Kosinski AS, Gaissert HA, et al. Minimally Invasive Versus Open Esophagectomy for Esophageal Cancer: A Comparison of Early Surgical Outcomes From The Society of Thoracic Surgeons National Database. Ann Thorac Surg 2016;101:1281-8; discussion 1288-9. 
14. In H, Palis BE, Merkow RP, et al. Doubling of 30-Day Mortality by 90 Days After Esophagectomy: A Critical Measure of Outcomes for Quality Improvement. Ann Surg 2016;263:286-291.

15. Rutegard M, Lagergren P, Johar A, et al. Time shift in early postoperative mortality after oesophagectomy for cancer. Ann Surg Oncol 2015;22:3144-3149.

16. Talsma AK, Lingsma HF, Steyerberg EW, et al. The 30-day versus in-hospital and 90-day mortality after esophagectomy as indicators for quality of care. Ann Surg 2014;260:267-273.

17. Messager M, Pasquer A, Duhamel A, et al. Laparoscopic Gastric Mobilization Reduces Postoperative Mortality After Esophageal Cancer Surgery: A French Nationwide Study. Ann Surg 2015;262:817-22; discussion 822-3.

18. Korhonen P, Malila N, Pukkala E, et al. The Finnish Cancer Registry as follow-up source of a large trial cohort--accuracy and delay. Acta Oncol 2002;41:381-388.

19. Lindblad M, Ye W, Lindgren A, et al. Disparities in the classification of esophageal and cardia adenocarcinomas and their influence on reported incidence rates. Ann Surg 2006;243:479485.

20. Sund R. Quality of the Finnish Hospital Discharge Register: a systematic review. Scand J Public Health 2012;40:505-515.

21. Ludvigsson JF, Andersson E, Ekbom A, et al. External review and validation of the Swedish national inpatient register. BMC Public Health 2011;11:450-2458-11-450. 
22. Quan H, Sundararajan V, Halfon P, et al. Coding algorithms for defining comorbidities in ICD-9-CM and ICD-10 administrative data. Med Care 2005;43:1130-1139.

23. Nilsson M, Kamiya S, Lindblad M, et al. Implementation of minimally invasive esophagectomy in a tertiary referral center for esophageal cancer. J Thorac Dis 2017;9:S817S825.

24. Kauppi J, Rasanen J, Sihvo E, et al. Open versus minimally invasive esophagectomy: clinical outcomes for locally advanced esophageal adenocarcinoma. Surg Endosc 2015;29:2614-2619.

25. Rouvelas I, Zeng W, Lindblad M, et al. Survival after surgery for oesophageal cancer: a population-based study. Lancet Oncol 2005;6:864-870.

26. Kassin MT, Owen RM, Perez SD, et al. Risk factors for 30-day hospital readmission among general surgery patients. J Am Coll Surg 2012;215:322-330.

27. Brusselaers N, Mattsson F, Lagergren J. Hospital and surgeon volume in relation to longterm survival after oesophagectomy: systematic review and meta-analysis. Gut 2014;63:13931400.

28. Zhou C, Zhang L, Wang H, et al. Superiority of Minimally Invasive Oesophagectomy in Reducing In-Hospital Mortality of Patients with Resectable Oesophageal Cancer: A MetaAnalysis. PLoS One 2015; 10:e0132889.

29. Yerokun BA, Sun Z, Jeffrey Yang CF, et al. Minimally Invasive Versus Open Esophagectomy for Esophageal Cancer: A Population-Based Analysis. Ann Thorac Surg 2016;102:416-423. 
30. Parameswaran R, Blazeby JM, Hughes R, et al. Health-related quality of life after minimally invasive oesophagectomy. Br J Surg 2010;97:525-531.

31. Maas KW, Cuesta MA, van Berge Henegouwen MI, et al. Quality of Life and Late Complications After Minimally Invasive Compared to Open Esophagectomy: Results of a Randomized Trial. World J Surg 2015;39:1986-1993. 


\section{Figure legends:}

Figure 1 A Kaplan-Meier curves of 90-day survival stratified by minimally invasive esophagectomy and open esophagectomy. 
Table 1 Characteristics of the 1614 study patients that underwent esophagectomy for esophageal cancer in Finland and Sweden in 2007-2014.

\begin{tabular}{|c|c|c|c|}
\hline Variable & $\begin{array}{l}\text { All operations } \\
\text { Patients }(\%) \\
n=1614\end{array}$ & $\begin{array}{c}\text { Open } \\
\text { esophagectomy } \\
\text { Patients }(\%) \\
\text { n=1397 }\end{array}$ & $\begin{array}{l}\text { Minimally invasive } \\
\text { esophagectomy } \\
\text { Patients (\%) } \\
\text { n=217 }\end{array}$ \\
\hline Mean age (SD) & $64.8(9.4)$ & $64.8(9.3)$ & $64.9(9.6)$ \\
\hline \multicolumn{4}{|l|}{ Sex } \\
\hline Male & $1289(80)$ & $1117(80)$ & $172(79)$ \\
\hline Female & $325(20)$ & $280(20)$ & $45(21)$ \\
\hline \multicolumn{4}{|l|}{$\begin{array}{l}\text { Charlson } \\
\text { comorbidity score }\end{array}$} \\
\hline 0 & $851(53)$ & $711(51)$ & $140(65)$ \\
\hline 1 & $278(17)$ & $251(18)$ & $27(12)$ \\
\hline 2 & $178(11)$ & $159(11)$ & $19(9)$ \\
\hline $3-5$ & $115(7)$ & $102(7)$ & $13(6)$ \\
\hline$\geq 6$ & $192(12)$ & $174(12)$ & $18(8)$ \\
\hline \multicolumn{4}{|l|}{ Tumor histology ${ }^{1}$} \\
\hline Adenocarcinoma & $1199(74)$ & $1031(74)$ & $168(77)$ \\
\hline $\begin{array}{l}\text { Squamous cell } \\
\text { carcinoma }\end{array}$ & $372(26)$ & $333(26)$ & $39(23)$ \\
\hline \multicolumn{4}{|l|}{ Year of surgery } \\
\hline 2007 & $185(11)$ & $184(13)$ & $1(0)$ \\
\hline 2008 & $174(11)$ & $168(12)$ & $6(3)$ \\
\hline 2009 & $196(12)$ & $182(13)$ & $14(6)$ \\
\hline 2010 & $218(14)$ & 189 (14) & $29(13)$ \\
\hline 2011 & $211(13)$ & $187(13)$ & $24(11)$ \\
\hline 2012 & 209 (13) & $178(13)$ & $31(14)$ \\
\hline 2013 & $239(15)$ & $197(14)$ & $42(19)$ \\
\hline $2014^{2}$ & $182(11)$ & $112(8)$ & $70(32)$ \\
\hline 30-day mortality & $31(1.9)$ & $27(1.9)$ & $4(1.8)$ \\
\hline 90-day mortality & $104(6.4)$ & $95(6.8)$ & $9(4.1)$ \\
\hline $\begin{array}{l}\text { Readmission rate } \\
\text { within } 30 \text { days }^{3}\end{array}$ & $183(11.6)$ & $164(12.0)$ & $19(8.9)$ \\
\hline $\begin{array}{l}\text { Readmission rate } \\
\text { within } 90 \text { days }^{4}\end{array}$ & $498(33.0)$ & $438(33.6)$ & $60(28.8)$ \\
\hline $\begin{array}{l}\text { Median hospital } \\
\text { stay, days (IQR) }\end{array}$ & $16(11)$ & $16(11)$ & $15(7)$ \\
\hline \multirow{2}{*}{\multicolumn{4}{|c|}{$\begin{array}{l}{ }^{1} \text { In a total of } 43 \text { cases no specific histology of the cancer was reported, or histology could not be } \\
\text { determined (undefined carcinoma). IQR, Interquartile range; SD, standard deviation } \\
{ }^{2} \text { Included operations from January 1, } 2014 \text { to October 2, } 2014\end{array}$}} \\
\hline & & & \\
\hline \multicolumn{4}{|c|}{${ }^{3}$ Patients who died during first 30 days after surgery were censored } \\
\hline${ }^{4}$ Patients who died do & ïrst 90 days after & $\mathrm{y}$ were censored & \\
\hline
\end{tabular}


Table 2 Hazard ratios (HRs) with 95\% confidence intervals (CI) of mortality comparing minimally invasive and open esophagectomy (reference group) for esophageal cancer within 90 and 30 days of surgery.

\begin{tabular}{cllll}
\hline & $\begin{array}{l}\text { Patient } \\
\text { Number }\end{array}$ & $\begin{array}{l}\text { Surgical approach } \\
\text { Open esophagectomy } \\
\text { HR (95\% CI) }\end{array}$ & $\begin{array}{l}\text { Minimally invasive esophagectomy } \\
\text { HR (95\% CI) }\end{array}$ & $P$-value \\
\hline $\begin{array}{c}\text { 90-day mortality } \\
\text { Crude }\end{array}$ & 1614 & 1 (reference) & $0.61(0.31-1.20)$ & 0.152 \\
$\begin{array}{c}\text { Adjusted } \\
\text { 30-day mortality }\end{array}$ & 1614 & 1 (reference) & $0.49(0.24-0.99)$ & 0.048 \\
$\quad \begin{array}{l}\text { Crude } \\
\text { Adjusted }\end{array}$ & 1614 & 1 (reference) & $0.96(0.34-2.74)$ & 0.936 \\
\hline
\end{tabular}

${ }^{1}$ Adjustment for age, sex, Charlson comorbidity index, histological type, year of surgery, and country. 
Table $3 \beta$ coefficient with standard error (SE) for comparison of length of hospital stay after minimally invasive (median 15 days) and open esophagectomy (median 16 days, reference group) for esophageal cancer.

\begin{tabular}{cllll}
\hline & $\begin{array}{l}\text { Patient } \\
\text { Number }\end{array}$ & $\begin{array}{l}\text { Surgical approach } \\
\text { Open esophagectomy } \\
\boldsymbol{\beta} \text { (SE) }\end{array}$ & $\begin{array}{l}\text { Minimally invasive esophagectomy } \\
\boldsymbol{\beta} \text { (SE) }\end{array}$ & $\boldsymbol{P}$-value \\
\hline $\begin{array}{c}\text { Hospital stay } \\
\text { Crude }\end{array}$ & 1553 & 1 (reference) & $-0.02(0.07)$ & 0.825 \\
Adjusted $^{2}$ & 1553 & 1 (reference) & $-0.17(0.08)$ & 0.030 \\
\hline
\end{tabular}

${ }^{1}$ Patients who died during hospital admission for esophagectomy were censored from the analysis

${ }^{2}$ Adjustment for age, sex, Charlson comorbidity index, histological type, year of surgery, and country 
Table 4 Hazard ratios (HRs) with 95\% confidence intervals (CI) of readmissions comparing minimally invasive and open esophagectomy (reference group) for esophageal cancer within 30 and 90 days of surgery.

\begin{tabular}{|c|c|c|c|c|}
\hline & $\begin{array}{l}\text { Patient } \\
\text { Number }\end{array}$ & $\begin{array}{l}\text { Surgical approach } \\
\text { Open esophagectomy } \\
\text { HR }(95 \% \text { CI })\end{array}$ & $\begin{array}{l}\text { Minimally invasive esophagectomy } \\
\text { HR (95\% CI) }\end{array}$ & $P$-value \\
\hline \multicolumn{5}{|c|}{ 30-day readmissions ${ }^{1}$} \\
\hline Crude & 1583 & 1 (reference) & $0.70(0.44-1.13)$ & 0.141 \\
\hline Adjusted $^{2}$ & 1583 & 1 (reference) & $0.57(0.34-0.94)$ & 0.028 \\
\hline \multicolumn{5}{|c|}{ 90-day readmissions ${ }^{3}$} \\
\hline Crude & 1510 & 1 (reference) & $0.81(0.61-1.07)$ & 0.138 \\
\hline Adjusted $^{2}$ & 1510 & 1 (reference) & $0.82(0.61-1.10)$ & 0.191 \\
\hline
\end{tabular}

${ }^{1}$ Patients who died during first 30 days after surgery were censored from the analysis

${ }^{2}$ Adjustment for age, sex, Charlson comorbidity index, histological type, year of surgery, and country

${ }^{3}$ Patients who died during first 90 days after surgery were censored from the analysis 


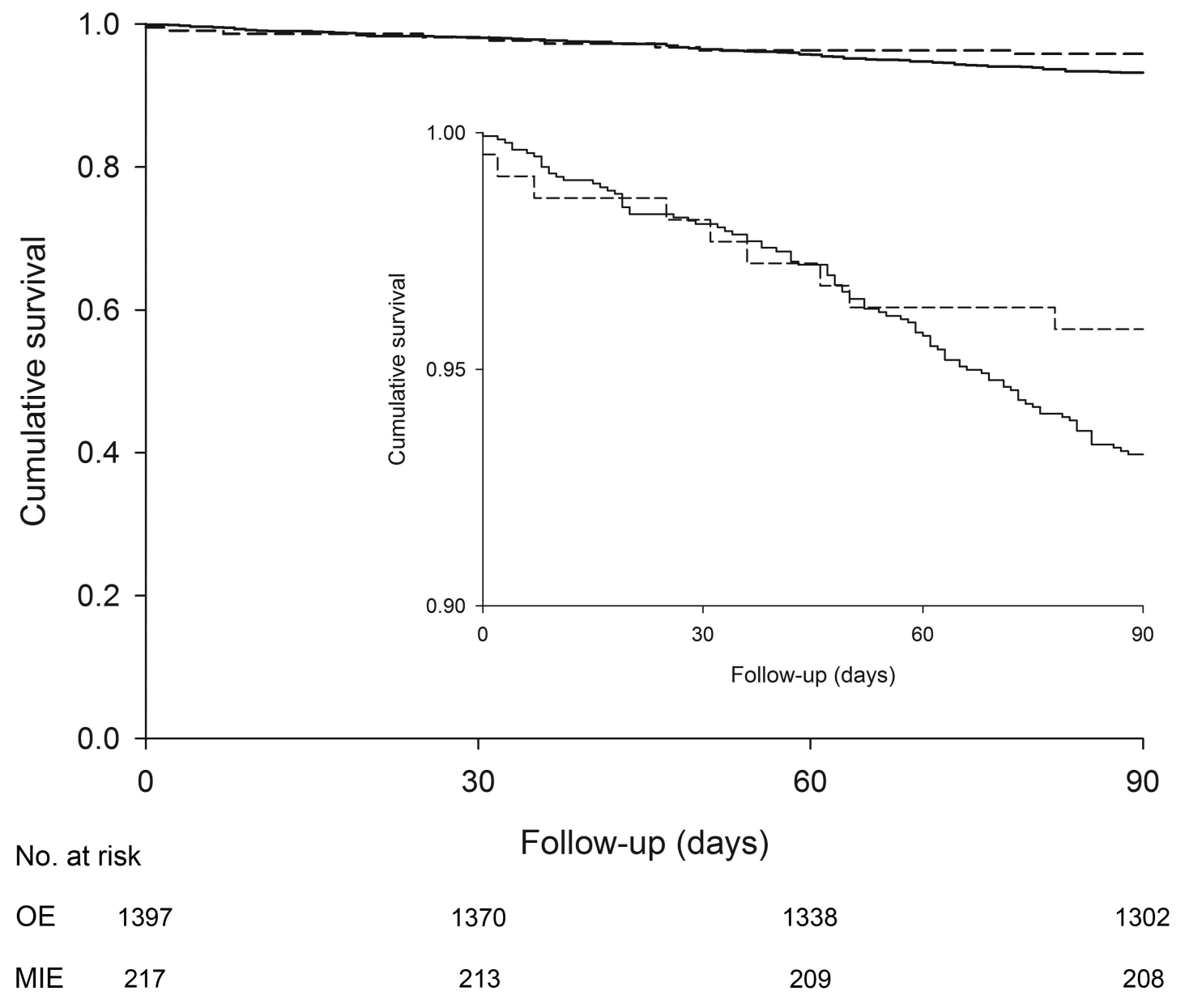

